\title{
Wall energy and wall thickness of exchange-coupled rare-earth transition-metal triple layer stacks
}

\author{
D. Raasch \\ Philips Research, Weißhausstrasse 2, D-52066 Aachen, Germany \\ C. Mathieu \\ University Kaiserslautern, Erwin-Schrödinger-Strasse 56, D-67663 Kaiserslautern, Germany
}

(Received 12 December 1996; accepted for publication 12 May 1997)

\begin{abstract}
The room-temperature wall energy $\sigma_{w}=4.0 \times 10^{-3} \mathrm{~J} / \mathrm{m}^{2}$ of an exchange-coupled $\mathrm{Tb}_{19.6} \mathrm{Fe}_{74.7} \mathrm{Co}_{5.7} / \mathrm{Dy}_{28.5} \mathrm{Fe}_{43.2} \mathrm{Co}_{28.3}$ double layer stack can be reduced by introducing a soft magnetic intermediate layer in between both layers exhibiting a significantly smaller anisotropy compared to $\mathrm{Tb}-\mathrm{FeCo}$ and $\mathrm{Dy}-\mathrm{FeCo} . \sigma_{w}$ will decrease linearly with increasing intermediate layer thickness, $d_{\mathrm{IL}}$, until the wall is completely located within the intermediate layer for $d_{\mathrm{IL}} \geqslant d_{w}$, where $d_{w}$ denotes the wall thickness. Thus, $d_{w}$ can be obtained from the plot $\sigma_{w}$ versus $d_{\mathrm{IL}}$. We determined $\sigma_{w}$ and $d_{w}$ on $\mathrm{Gd}-\mathrm{FeCo}$ intermediate layers with different anisotropy behavior (perpendicular and in-plane easy axis) and compared the results with data obtained from Brillouin light-scattering measurements, where exchange stiffness, $A$, and uniaxial anisotropy, $K_{u}$, could be determined. With the knowledge of $A$ and $K_{u}$, wall energy and thickness were calculated and showed an excellent agreement with the magnetic measurements. A ten times smaller perpendicular anisotropy of $\mathrm{Gd}_{28.1} \mathrm{Fe}_{71.9}$ in comparison to $\mathrm{Tb}-\mathrm{FeCo}$ and $\mathrm{Dy}-\mathrm{FeCo}$ resulted in a much smaller $\sigma_{w}=1.1 \times 10^{-3} \mathrm{~J} / \mathrm{m}^{2}$ and $d_{w}=24 \mathrm{~nm}$ at $300 \mathrm{~K}$. A Gd $\mathrm{Gd}_{34.1} \mathrm{Fe}_{61.4} \mathrm{Co}_{4.5}$ with in-plane anisotropy at room temperature showed a further reduced $\sigma_{w}=0.3 \times 10^{-3} \mathrm{~J} / \mathrm{m}^{2}$ and $d_{w}=17 \mathrm{~nm}$. The smaller wall energy was a result of a different wall structure compared to perpendicular layers. (C) 1997 American Institute of Physics. [S0021-8979(97)02616-9]
\end{abstract}

\section{INTRODUCTION}

Magneto-optical (MO) single layer disks based on amorphous rare-earth (RE) transition-metal (TM) alloys such as $\mathrm{Tb}-\mathrm{FeCo}$ and $\mathrm{Dy}-\mathrm{FeCo}$ exhibit very good recording properties such as high carrier-to-noise ratio, and low required write/erase laser powers, and fields. ${ }^{1,2}$ The storage capacity of current MO disks has been increased by a factor of 4 compared to the first products. Due to several different approaches regarding recorder hardware, data processing, storage formats, and the use of exchange-coupled MO layers, this development will continue, and a disk capacity that is sufficient for consumer audio and video applications will be achieved in the near future. ${ }^{3,4}$

The interface wall energy $\sigma_{w}$ determines the switching fields of an exchange-coupled double layer stack. One can adjust the wall energy independently from the magnetic properties of the other layers by introducing an additional intermediate layer. The reduction of $\sigma_{w}$ depends on intermediate layer thickness $d_{\mathrm{IL}}$, and the fraction of the wall located within the intermediate layer. The latter depends on the intrinsic wall thickness $d_{w}$ of an intermediate layer, which is a function of anisotropy and exchange stiffness. A $\mathrm{Gd}_{28.1} \mathrm{Fe}_{71.9}$ with a perpendicular anisotropy and a $\mathrm{Gd}_{34.1} \mathrm{Fe}_{61.4} \mathrm{Co}_{4.5}$ with an in-plane anisotropy at room temperature were tested as intermediate layers. $\sigma_{w}$ and $d_{w}$ were determined as a function of intermediate layer thickness. Exchange stiffness and anisotropy of $\mathrm{Gd}-\mathrm{Fe}$ single layer samples were determined by Brillouin light-scattering and magnetic torque measurements. From these results, also $\sigma_{w}$ and $d_{w}$ were calculated and compared to the results of triple layer stacks.

\section{SAMPLE PREPARATION AND EXPERIMENTAL CONDITIONS}

The exchange-coupled films were deposited by dcmagnetron sputtering. The cathodes were mounted in a Balzers BAK 600 deposition chamber with a target-substrate distance between 6.6 and $7.9 \mathrm{~cm}$. The substrates were mounted on a holder rotating at $1 \mathrm{~Hz}$, and were moved alongside the cathode during deposition to obtain a homogeneous composition and layer thickness. The $\mathrm{Tb}_{19.6} \mathrm{Fe}_{74.7} \mathrm{Co}_{5.7} /$ $\mathrm{Dy}_{28.5} \mathrm{Fe}_{43.2} \mathrm{Co}_{28.3}$ double layers were sputtered at an argon pressure of $0.55 \mathrm{~Pa}$ and a power density (PD) of $1.3 \mathrm{~W} / \mathrm{cm}^{2}$. The triple layer stacks have additional intermediate layers $\mathrm{Gd}_{28.1} \mathrm{Fe}_{71.9}\left(P_{\mathrm{Ar}}=1.2 \mathrm{~Pa}, P D=1.0 \mathrm{~W} / \mathrm{cm}^{2}\right)$ and $\mathrm{Gd}_{34.1} \mathrm{Fe}_{61.4} \mathrm{Co}_{4.5}\left(P_{\mathrm{Ar}}=2.1 \mathrm{~Pa}, P D=2.0 \mathrm{~W} / \mathrm{cm}^{2}\right)$. The background pressure was $2 \times 10^{-5} \mathrm{~Pa}$. The MO layer stack was coated for chemical protection purposes with a Ti reflective layer of $26 \mathrm{~nm}$ thickness. The composition was determined by electron-probe microanalysis.

All samples were magnetically characterized between 90 and $600 \mathrm{~K}$, yielding saturation magnetization $M_{s}(T)$, coercivity $H_{c}(T)$, and switching fields $H^{ \pm}$of exchange-coupled multilayer stacks. $M_{s}$ and $H_{c}$ were measured with a vibrating sample magnetometer; $H^{ \pm}$were determined with a Hall magnetometer. The anisotropy $K_{u}(T)$ was measured with a torque magnetometer. However, the method is only applicable to single layers. Compensation temperature $T_{\text {comp }}$ and Curie temperature $T_{C}$ were derived from the magnetic data.

The Brillouin light-scattering (BLS) experiments were performed on $\mathrm{Gd}_{28.1} \mathrm{Fe}_{71.9}$ layers with thicknesses between 20 and $50 \mathrm{~nm}$. The layers were covered with a $200 \mathrm{~nm} \mathrm{Al}$ layer to protect the samples from thermal overheating in the 
laser spot. The laser light of about $30 \mathrm{~mW}$ was focused onto the film through the transparent substrate. A magnetic field of about $2300 \mathrm{kA} / \mathrm{m}$ was applied parallel to the film plane to ensure that the layers were in-plane magnetized. The BLS measurements provide data of the exchange stiffness $A$ and the gyromagnetic factor $g_{\text {eff }}$ at room temperature.

\section{RESULTS AND DISCUSSION}

\section{A. Brillouin light scattering}

Brillouin light scattering is the measurement of the inelastically scattered light from dynamic excitations, which in the present case, are the so-called dipolar Damon-Eshbach mode and the standing spin-wave modes. The DamonEshbach mode is a surface mode, and its frequency depends on the magnetization and anisotropy contributions. Standing spin-wave modes are modes with their wave vector quantized perpendicular to the film, and their frequencies depend in addition on the exchange stiffness constant, $A$. For these modes, apart from the crossing regime with the DamonEshbach mode, the frequencies show a characteristic decrease proportional to the inverse of the square of the layer thickness. In our experiments, the Damon-Eshbach mode, as well as the first and second standing spin-wave modes, could be observed. A fit to the experimentally obtained spin-wave frequencies was performed within the framework of a suitable model, which includes all details of spin-wave dispersion. ${ }^{5}$ The exchange stiffness, $A$, and the effective Landé factor, $g_{\text {eff }}$, were used as fit parameters. $g_{\text {eff }}$ is a weighted average of the $g$ factors of the Fe and Gd sublattices, and is defined by the equation for the gyromagnetic ratio, $\gamma=1 / 2 \gamma_{e} g_{\text {eff }}$, where $\gamma_{e}$ is the free-electron value. The uniaxial anisotropy $K_{u}=0.34 \times 10^{5} \mathrm{~J} / \mathrm{m}^{3}$ and the saturation magnetization $M_{s}=102 \mathrm{kA} / \mathrm{m}$, were used as input parameters. The data were obtained from magnetic measurements on sputtered $\mathrm{Gd}_{28.1} \mathrm{Fe}_{71.9}$ single layer samples. For the exchange stiffness, we obtained $A=(1.7 \pm 0.5) \times 10^{-12} \mathrm{~J} / \mathrm{m}$, and for the effective Lande factor $g_{\text {eff }}=1.3 \pm 0.3$. The value for $A$ is in agreement with results of the systems $\mathrm{Gd}-\mathrm{Co}$, $\mathrm{GdTb}-\mathrm{Fe}$, and $\mathrm{GdNd}-\mathrm{Fe}^{6,7}$ A literature value for $\mathrm{Gd}_{33} \mathrm{Fe}_{67}$ (Ref. 8) of $A=3.6 \times 10^{-12} \mathrm{~J} / \mathrm{m}$ seems to be too large. The effective Landé factor is in good agreement with data reported by $\mathrm{Ng}$ et al. ${ }^{9}$ The exchange stiffness $A$ mainly depends on the Curie temperature $T_{C}$, if very similar RE-TM alloys are compared. ${ }^{10} \mathrm{Gd}_{28.1} \mathrm{Fe}_{71.9}$ and $\mathrm{Gd}_{34.1} \mathrm{Fe}_{61.4} \mathrm{Co}_{4.5}$ have very similar $T_{C}$ of 515 and $500 \mathrm{~K}$, respectively. Therefore $A=(1.7 \pm 0.5) \times 10^{-12} \mathrm{~J} / \mathrm{m}$ can also be assumed for $\mathrm{Gd}-\mathrm{FeCo}$. From exchange stiffness and anisotropy, the wall energy and wall thickness of the investigated layers can be calculated, see the next section.

\section{B. Magnetic measurements}

The switching fields for a magnetic reversal of a layer as part of an exchange-coupled double layer stack differs from $H_{c}$ of the same layer, if single and uncoupled. The reversal of each individual layer will lead to the creation $(+)$ or annihilation (-) of an interface wall, as shown in Fig. 1 for a $\mathrm{Tb}-\mathrm{FeCo} / \mathrm{Dy}-\mathrm{FeCo}$ double layer at $350 \mathrm{~K}$. The interface

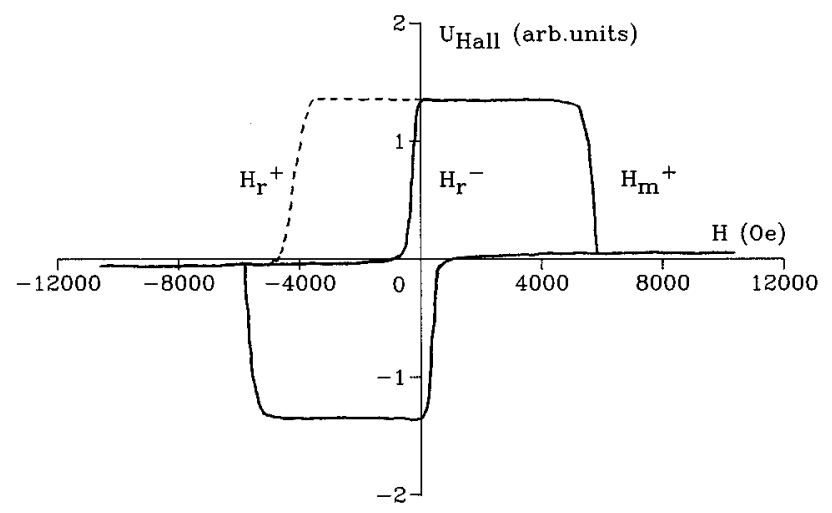

FIG. 1. Hysteresis loop of a $\mathrm{Tb}_{19.6} \mathrm{Fe}_{74.7} \mathrm{Co}_{5.7} / \mathrm{Dy}_{28.5} \mathrm{Fe}_{28.5} \mathrm{Co}_{28.3}$ double layer sputtered at an argon pressure of $0.55 \mathrm{~Pa}$ and a power density of $1.3 \mathrm{~W} / \mathrm{cm}^{2}$. The layer thicknesses were 52 and $100 \mathrm{~nm}$, respectively. The dashed loop represents the minor loop of the Dy-FeCo layer. The fields $H^{ \pm}$represent the switching fields for creation $(+)$and annihilation $(-)$of an interface wall. The indices $(m)$ and $(r)$ denote the $\mathrm{Tb}-\mathrm{FeCo}$ and $\mathrm{Dy}-\mathrm{FeCo}$ layers, respectively.

wall energy $\sigma_{w}$ resulted from the difference between the switching fields of the minor loop $\mathrm{H}^{+}$for creation and $\mathrm{H}^{-}$ for annihilation of an interface wall ${ }^{11}$

$$
\sigma_{w}=\mu_{0} M_{s} d\left(H^{+}-H^{-}\right),
$$

where $M_{s}$ and $d$ denote the saturation magnetization and thickness of the switched Dy-FeCo layer. For $M_{s}$ $=80 \mathrm{kA} / \mathrm{m}$ and $d=100 \mathrm{~nm}$, we determined a wall energy of $3.1 \times 10^{-3} \mathrm{~J} / \mathrm{m}^{2}$ at $350 \mathrm{~K}$. At $300 \mathrm{~K}$, the $\mathrm{Tb}-\mathrm{FeCo}$ could not be switched due to its large coercivity, but the determined minor loop of the Dy-FeCo layer revealed a wall energy of $4.0 \times 10^{-3} \mathrm{~J} / \mathrm{m}^{2}$.

The triple layer stacks have one additional soft magnetic layer (intermediate layer) between the $\mathrm{Tb}-\mathrm{FeCo}$ and $\mathrm{Dy}-$ FeCo layers. Then, the wall energy also depends on magnetic properties and the thickness of the intermediate layer. The tested intermediate layers showed a smaller anisotropy compared to $\mathrm{Tb}-\mathrm{FeCo}$ and $\mathrm{Dy}-\mathrm{FeCo}$. Because the wall energy is a function of anisotropy, it will decrease if the wall is located within a layer with a smaller anisotropy. The contribution of the intermediate layer to the total wall energy depends on its layer thickness $d_{\mathrm{IL}}$. A thicker intermediate layer contains a larger fraction of the interface wall resulting in a decrease of $\sigma_{w}$ for increasing $d_{\mathrm{IL}}$. If $d_{\mathrm{IL}}$ is larger than the wall thickness $d_{w}$, the wall will be totally located in the intermediate layer. Then $\sigma_{w}$ cannot be reduced further, and the measured $\sigma_{w}$ corresponds to the intrinsic wall energy of the intermediate layer, and it will be independently from $d_{\mathrm{IL}}$ for $d_{\mathrm{IL}} \geqslant d_{w}$. The thickness $d$, where the decrease of $\sigma_{w}$ stops, corresponds to the wall thickness $d_{w}$.

The tested intermediate layers $\mathrm{Gd}_{28.1} \mathrm{Fe}_{71.9}\left(T_{C}=515 \mathrm{~K}\right.$, $\left.T_{\text {comp }}=390 \mathrm{~K}\right)$ and $\mathrm{Gd}_{34.1} \mathrm{Fe}_{61.4} \mathrm{Co}_{4.5} \quad\left(T_{C}=500 \mathrm{~K}, T_{\text {comp }}\right.$ $>T_{C}$ ) showed different anisotropy behaviors. For $\mathrm{Gd}_{28.1} \mathrm{Fe}_{71.9}$, we determined a perpendicular anisotropy $K_{u}=0.34 \times 10^{5} \mathrm{~J} / \mathrm{m}^{3}$ at $300 \mathrm{~K}$, as shown in Fig. 2. This is approximately one order of magnitude smaller in comparison 


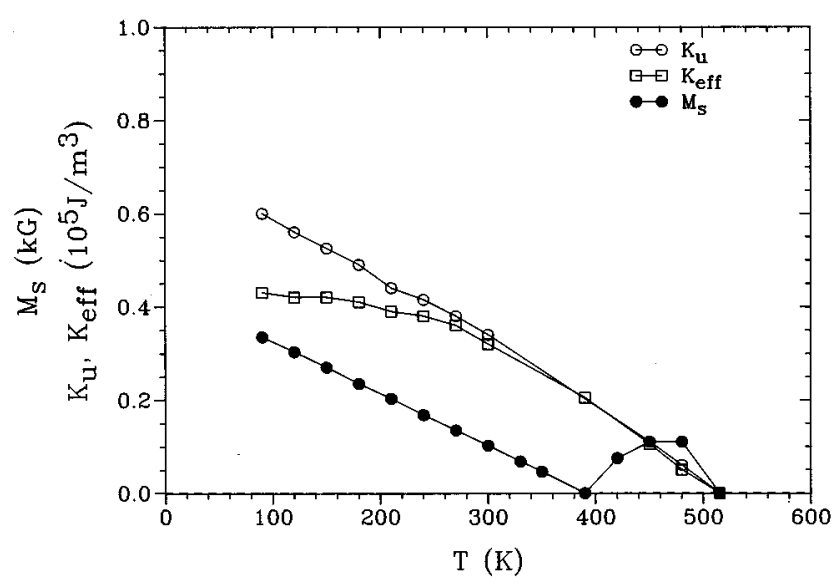

FIG. 2. Uniaxial anisotropy $K_{u}$, effective anisotropy $K_{\text {eff }}$, and saturation magnetization $M_{s}$ of a $\mathrm{Gd}_{28.1} \mathrm{Fe}_{71.9}$ single layer (thickness $50 \mathrm{~nm}$ ) as function of temperature.

to $\mathrm{Tb}-\mathrm{FeCo}$ and $\mathrm{Dy}-\mathrm{FeCo},{ }^{6,10}$ and corresponds to measurements on evaporated $\mathrm{Gd}_{1-x} \mathrm{Fe}_{x}$ layers, where a perpendicular anisotropy was found for $0.70<x<0.82$. $^{12}$

Evaporated $\mathrm{Gd}_{1-x} \mathrm{Co}_{x}$ alloys showed allways in-plane anisotropy. The addition of certain amount of Co to Gd-rich $\mathrm{Gd}-\mathrm{Fe}$ alloys causes in-plane anisotropy at room temperature. On evaporated Gd-FeCo alloys, no perpendicular anisotropy was found for $\mathrm{Co} / \mathrm{Fe}$ ratios larger than 0.47 . Samples with a smaller $\mathrm{Co} / \mathrm{Fe}$ ratio showed a temperature range with in-plane anisotropy, which was a function of $\mathrm{Gd}$ content. $^{12}$ For sputtered samples, we also expected an inplane anisotropy at a suitable Co content. $\mathrm{Gd}_{34.1} \mathrm{Fe}_{61.4} \mathrm{Co}_{4.5}$ showed in-plane anisotropy below $335 \mathrm{~K}$, where a large saturation magnetization led to a negative $K_{\text {eff }}=K_{u}-\mu_{0} / 2 M_{s}^{2}$ despite a $K_{u}>0$, as shown in Fig. 3. For $T>335 \mathrm{~K}$, the uniaxial anisotropy $K_{u}$ is larger than $\mu_{0} / 2 M_{s}^{2}$, resulting in a perpendicular anisotropy. For the in-plane intermediate layer, the magnetic moments at the middle of the interface wall are aligned parallel to the easy axis of the intermediate layer. Therefore, we expected a different function $\sigma_{w}\left(d_{\mathrm{IL}}\right)$ for $\mathrm{Gd}-\mathrm{FeCo}$ intermediate layers in comparison to $\mathrm{Gd}-\mathrm{Fe}$ layers with perpendicular anisotropy.

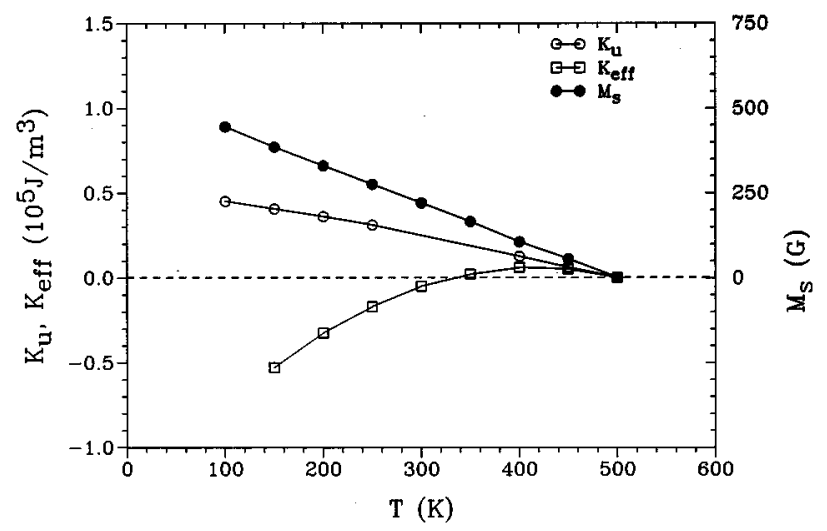

FIG. 3. Uniaxial anisotropy $K_{u}$, effective anisotropy $K_{\text {eff }}$, and saturation magnetization $M_{s}$ of a $\mathrm{Gd}_{34.1} \mathrm{Fe}_{61.4} \mathrm{Co}_{4.5}$ single layer (thickness $51 \mathrm{~nm}$ ) as function of temperature.

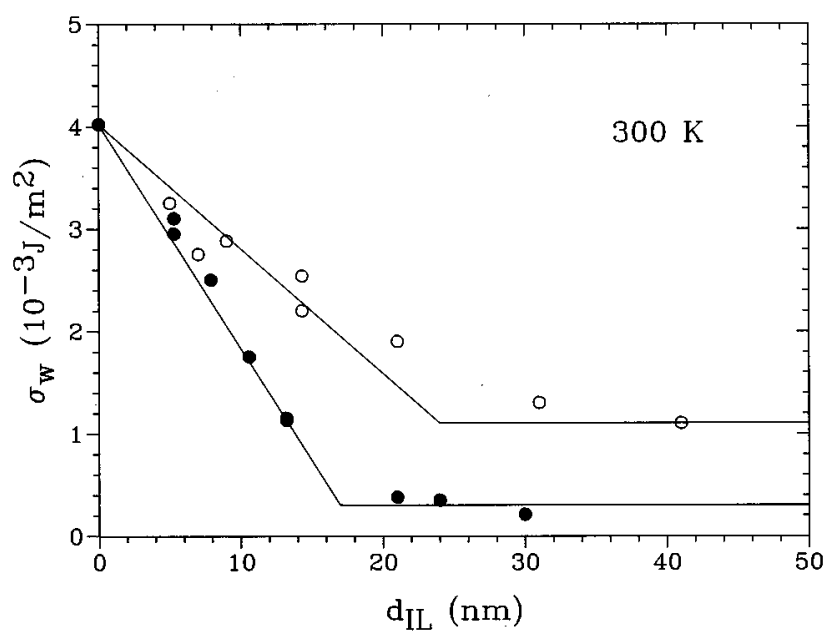

FIG. 4. Wall energy $\sigma_{w}$ as a function of intermediate layer thickness $d_{\mathrm{IL}}$ for (O) $\mathrm{Gd}_{28.1} \mathrm{Fe}_{71.9}$ and (O) $\mathrm{Gd}_{34.1} \mathrm{Fe}_{61.4} \mathrm{Co}_{4.5}$ at $300 \mathrm{~K}$. The thickness of $\mathrm{Tb}-$ FeCo and Dy-FeCo layers was 22.0 and $50.4 \mathrm{~nm}$, respectively. The solid lines were calculated from BLS data using Eqs. (2)-(4).

Wall energy and wall thickness are determined by the minimum of the total wall energy $\int\left(E_{\mathrm{ex}}+E_{K}+E_{H}+E_{D}\right) d z$ = minimum, where $E_{\mathrm{ex}}, E_{k}, E_{H}$, and $E_{D}$ denote the exchange energy, anisotropy energy, field energy, and demagnetizing energy, respectively. $E_{H} / K_{u}$ is smaller than 0.2 for $(\mathrm{Gd}, \mathrm{Tb})-(\mathrm{Fe}, \mathrm{Co})$ and $(\mathrm{Gd}, \mathrm{Dy})-(\mathrm{Fe}, \mathrm{Co})$ layers. ${ }^{6,10}$ This is more than ever valid for corrections due to the demagnetizing energy scaling with $M_{s}^{2}$, which is much less than $H^{ \pm} M_{s}$. In this case, walls can be treated as classic $180^{\circ}$ Bloch walls. ${ }^{13}$ Then, wall energy $\sigma_{w}$ and thickness $d_{w}$ are given by ${ }^{14}$

$$
\sigma_{w}=4 \sqrt{A K_{u}} \quad d_{w}=\pi \sqrt{A / K_{u}} .
$$

Both equations can be applied for double and triple layer stacks, if all layers exhibit perpendicular anisotropy, i.e., $\mathrm{Gd}-\mathrm{Fe}$ intermediate layers. Figure 4 shows the wall energy as a function of intermediate layer thickness $d_{\mathrm{IL}}$ for triple layer measurements and data calculated from Brillouin lightscattering measurements. The result for exchange-coupled double layers corresponds to the wall energy of $\mathrm{Tb}-\mathrm{FeCo}$ and Dy-FeCo single layers, assuming typical values for exchange stiffness $A$ and anisotropy $K_{u}$. With $A=3.1$ $\times 10^{-12} \mathrm{~J} / \mathrm{m}$ and $K_{u}=3.2 \times 10^{5} \mathrm{~J} / \mathrm{m}^{3}, 6,10$ a wall energy of $4.0 \times 10^{-3} \mathrm{~J} / \mathrm{m}^{2}$ was expected, which was in an excellent agreement with the magnetic measurement. For $\mathrm{Gd}_{28.1} \mathrm{Fe}_{71.9}$, a minimum wall energy of $1.1 \pm 0.1$ $\times 10^{-3} \mathrm{~J} / \mathrm{m}^{2}$ was obtained for intermediate layer thicknesses $d_{\mathrm{IL}} \geqslant d_{w}$. From BLS data $\left[K_{u}=0.34 \times 10^{5} \mathrm{~J} / \mathrm{m}^{3}\right.$ and $A$ $\left.=(1.7 \pm 0.5) \times 10^{-12} \mathrm{~J} / \mathrm{m}\right]$, a wall energy of $(1.0 \pm 0.2)$ $\times 10^{-3} \mathrm{~J} / \mathrm{m}^{2}$ was calculated using Eq. (2). The triple layer measurements revealed a wall thickness $d_{w}$ of $24 \pm 2 \mathrm{~nm}$. A wall thickness of $22 \pm 3 \mathrm{~nm}$ was determined from BLS data. Both results were in excellent agreement.

In the case of intermediate layers with in-plane anisotropy, the wall structure is not a simple $180^{\circ}$ Bloch wall. The magnetic moments at the interface to the $\mathrm{Tb}-\mathrm{FeCo}$ and $\mathrm{Dy}-$ FeCo layers are aligned parallel to the perpendicular easy axis of the $\mathrm{Tb}-\mathrm{FeCo}$ and $\mathrm{Dy}-\mathrm{FeCo}$ layers. But the magnetic 
moments in the middle of the wall are parallel to the in-plane easy axis of the $\mathrm{Gd}_{34.1} \mathrm{Fe}_{61.4} \mathrm{Co}_{4.5}$ layer. Therefore, one can consider this type of wall as the sum of two $90^{\circ}$ walls, where the easy axis of both sides of a $90^{\circ}$ wall are perpendicular to each other. Lilley calculated solutions of $\int\left(E_{\mathrm{ex}}+E_{K}+E_{H}\right.$ $\left.+E_{D}\right) d z=$ minimum for walls in materials with two easy axes not parallel to each other. ${ }^{15}$ Then, the energy and thickness of $90^{\circ}$ walls are given by ${ }^{16}$

$$
\begin{aligned}
& \sigma_{w}(\mathrm{Gd}-\mathrm{FeCo})=2 \times \sigma_{w}\left(90^{\circ}\right)=2 \sqrt{A K_{u}}, \\
& d_{w}(\mathrm{Gd}-\mathrm{FeCo})=2 \times d_{w}\left(90^{\circ}\right)=\sqrt{8} \ln 2 \sqrt{A / K_{u}} .
\end{aligned}
$$

Inserting magnetically measured $K_{u}=0.25 \times 10^{5} \mathrm{~J} / \mathrm{m}^{3}$ and $A=(1.7 \pm 0.5) \times 10^{-12} \mathrm{~J} / \mathrm{m}$, obtained from BLS measurements, the wall energy and wall thickness of $\mathrm{Gd}-\mathrm{FeCo}$ triple layers should be $(0.4 \pm 0.1) \times 10^{-3} \mathrm{~J} / \mathrm{m}^{2}$ and (19 $\pm 3) \mathrm{nm}$, respectively. Switching field experiments on $\mathrm{Gd}-$ FeCo triple layers revealed an interface wall energy of $(0.3$ $\pm 0.1) \times 10^{-3} \mathrm{~J} / \mathrm{m}^{2}$ and a wall thickness of $(17 \pm 1) \mathrm{nm}$, see Fig. 4. Also, for $\mathrm{Gd}-\mathrm{FeCo}$, an excellent agreement between the BLS data and triple layer measurements was obtained. This also shows that the interface wall of the triple layer with an in-plane intermediate layer can be treated as a double $90^{\circ}$ wall.

The wall energy measurements of triple layer stacks with a varied thickness of intermediate layers enables the determination of the wall thickness of the used intermediate layer. The method can be applied to magnetic layers, which the anisotropy is significantly smaller than the $K_{u}$ of Tb-FeCo and Dy-FeCo of approximately $3.0 \times 10^{5} \mathrm{~J} / \mathrm{m}^{3}$. This applies to a large number of magnetic alloys. With this method, wall thicknesses down to $10 \mathrm{~nm}$ can be easily determined only using a hysteresis looper and a vibrating sample magnetometer. With other techniques, like optical or magnetic force microscopes, it is more difficult to obtain a resolution better than $50 \mathrm{~nm}$.

\section{ACKNOWLEDGMENTS}

The authors would like to thank G. Much and $\mathrm{H}$. Wierenga for the sample preparation and the magnetic measurements. The authors also thank B. Hillebrands for helpful discussions and support.

${ }^{1}$ D. Raasch, IEEE Trans. Magn. MAG-29, 34 (1993).

${ }^{2}$ D. Raasch, J. P. C. Bernards, C. Clausen, and G. Much, J. Magn. Magn. Mater. 148, 359 (1995).

${ }^{3}$ J. Daval and B. Bechevet, J. Magn. Magn. Mater. 129, 98 (1994).

${ }^{4}$ E. M. Engler, J. Magn. Soc. Jpn. 19, 1 (1995).

${ }^{5}$ B. Hillebrands, Phys. Rev. B 41, 530 (1990).

${ }^{6}$ D. Raasch, J. Reck, C. Mathieu, and B. Hillebrands, J. Appl. Phys. 76, 1145 (1994).

${ }^{7}$ C. Mathieu, B. Hillebrands, and D. Raasch, IEEE Trans. Magn. MAG-30, 4434 (1994).

${ }^{8}$ C. Vittoria, P. Lubitz, and V. Ritz, J. Appl. Phys. 49, 4908 (1978).

${ }^{9}$ W. Ng, R. F. Soohoo, and R. C. Taylor, J. Appl. Phys. 53, 2359 (1982).

${ }^{10}$ D. Raasch and J. Reck, J. Appl. Phys. 74, 1229 (1993).

${ }^{11}$ T. Kobayashi, H. Tsuji, S. Tsunashima, and S. Uchiyama, Jpn. J. Appl. Phys. 20, 2089 (1981).

${ }^{12}$ D. Raasch, H. Wierenga, and H. Borg, J. Magn. Soc. Jpn. 20, 199 (1996).

${ }^{13}$ J. E. L. Bishop, J. Phys. D 7, 2369 (1974).

${ }^{14}$ S. Chikazumi and S. H. Charap, Physics of Magnetism (Krieger, Malabar, FL, 1964).

${ }^{15}$ B. A. Lilley, Philos. Mag. 41, 792 (1950).

${ }^{16} \mathrm{~W}$. Döring, in Handbuch der Physik, edited by S. Flügge (Springer, Freiburg, 1966), Vol. XVIII/2, p. 385. 